\title{
Mixed versus pure variants of desmoplastic melanoma: a genetic and immunohistochemical appraisal
}

\author{
Daniel D Miller ${ }^{1}$, Andrew Emley ${ }^{1}$, Shi Yang ${ }^{2}$, Joanna E Richards ${ }^{1}$, Jung Eun Lee ${ }^{1}$, \\ April Deng ${ }^{3}$, Mai P Hoang ${ }^{4}$ and Meera Mahalingam ${ }^{1}$
}

${ }^{1}$ Dermatopathology Section, Department of Dermatology, Boston University School of Medicine, Boston, MA, USA; ${ }^{2}$ Department of Pathology, Boston University School of Medicine, Boston, MA, USA; ${ }^{3}$ Department of Pathology, UMASS Medical School, Worchester, MA, USA and ${ }^{4}$ Department of Pathology, Massachusetts General Hospital, Boston, MA, USA

\begin{abstract}
Desmoplastic melanoma is subclassified into pure and mixed variants with a higher rate of lymph node metastasis in the latter. Given that reasons for these biological differences are not currently known, we investigated these subtypes with techniques that included genetic and immunohistochemical analyses of 43 cases of desmoplastic melanoma (24 pure, 19 mixed). Direct DNA sequencing was performed on BRAFV600E, RET gene (coding region on exon 11) and KIT (exons 11, 13 and 17). Immunohistochemical stains were performed with antibodies to markers of significance with respect to biological potential of nevomelanocytic proliferations and/or desmoplastic melanoma (Ki-67, CD117, nestin, clusterin, SOX10 and CD271/p75NTR). Polymorphism at the RET coding region (RETp) was noted in $33 \%$ of pure $(8 / 24$ cases) versus $24 \%$ of mixed (4/17 cases); BRAFV600E was absent in all cases of pure (0/24 cases) versus $6 \%$ of mixed (1/17 cases); no mutations were found in any of the cases on analyses of exons 11,13 and 17 of the $c-K I T$ gene $(P=N S$ for all). For immunohistochemical analyses of pure versus mixed: mean percentage of $\mathrm{Ki}-67$ nuclear positivity was $5 \%$ (s.d. $=5.6$ ) versus $28 \%$ (s.d. $=12.6, P<0.001)$; CD117 stained $26 \%(6 / 23$ cases) versus $78 \%(14 / 18$ cases, $P<0.01)$; nestin stained $83 \%(n=19 / 23$ cases) versus $89 \%(16 / 18$ cases, $P=N S)$; clusterin stained $4 \%(1 / 23$ cases) versus $6 \%$ (1/18 cases, $P=N S)$; SOX10 87\% (20/23 cases) versus $94 \%$ (17/18 cases, $P=N S)$ and CD271 stained $61 \%(14 / 23$ cases) versus $67 \%(12 / 18$ cases, $P=N S)$. Increased CD117 staining in the mixed variant suggests that alterations in the KIT protein may be involved in tumor progression. In addition, the proliferative index of the mixed variant was higher than that of the pure variant.
\end{abstract}

Modern Pathology (2012) 25, 505-515; doi:10.1038/modpathol.2011.196; published online 9 December 2011

Keywords: BRAF; CD117; desmoplasia; DNA sequencing; KIT; melanoma; RET

Desmoplastic melanoma is a rare variant of invasive cutaneous melanoma, with an annual incidence rate of approximately 2 per $1000000 .{ }^{1}$ Features unique to this melanoma type include delayed diagnosis, deep invasion, increased perineural invasion and local recurrence. ${ }^{2,3}$ George et $a l^{4}$ recently reviewed 87 cases of desmoplastic melanoma and identified a

Correspondence: Professor M Mahalingam, MD, PhD, FRCPath, Dermatopathology Section, Department of Dermatology, Boston University School of Medicine, 609 Albany Street, J-301, Boston, MA 02118, USA.

E-mail: mmahalin@bu.edu

Received 29 August 2011; revised 17 October 2011; accepted 17 October 2011; published online 9 December 2011 significant difference in propensity for regional lymph node metastasis in the variant composed of a mixed population of cells, that is, cells resembling conventional melanoma in addition to spindled cells, compared with the variant composed predominantly of spindled cells, lending credence to the concept of subclassifying desmoplastic melanoma into mixed and pure subtypes.

Although several molecular pathways are important in the tumorigenic progression of melanoma, the RAS/MAP kinase pathway appears to be the most important in conventional types. Approximately $60 \%$ of conventional melanomas have $B R A F$ mutations, but genetic profiling studies indicate that desmoplastic melanoma does not exhibit mutations 
in $B R A F$ to this extent. ${ }^{5-9}$ Recently, the frequency of a polymorphism of RET, a receptor tyrosine kinase whose ligand is glial cell line-derived neurotrophic factor (GDNF), at codon G691S has been shown to be significantly increased in desmoplastic versus conventional melanoma, a finding which in part explains the more prominent neurotropism seen in the desmoplastic variant. ${ }^{10}$ Gene expression profiling has also demonstrated multiple genetic differences between desmoplastic and conventional melanoma; many of these differences cluster to specific phenotypic families, including melanin pigment synthesis and neurotrophic factors. ${ }^{11}$

Although several studies have demonstrated the genetic and immunohistochemical profile of desmoplastic melanoma, the histopathological subtypes (mixed and pure) have not been characterized in this manner. In the current study, we sought to characterize these two subtypes genetically and immunohistochemically. Gene sequencing studies included analyses of RET, BRAF and KIT genes. Rationale for the investigation of $R E T$ and $B R A F$ was based on previous results described above, in which these two genes have different expression in conventional and desmoplastic melanoma; the initial hypothesis was that they may also be differentially expressed in the pure and mixed desmoplastic subtypes. KIT was investigated on the basis of the rationale that melanomas lacking $B R A F$ mutations may have mutations or copy number alterations in $C$-KIT. ${ }^{12}$ Immunohistochemical markers included the following: nestin, an intermediate filament protein, a melanocyte stem cell marker and a marker for melanoma-initiating cells, associated with poor prognosis in conventional melanoma; ${ }^{13}$ clusterin, an $80-\mathrm{kDa}$ glycoprotein of undefined biological significance shown to be significantly increased in desmoplastic melanoma; ${ }^{11}$ SOX10, a neural crest stem cell marker, expressed in melanocytes, specifically, strongly expressed by desmoplastic melanomas; ${ }^{14}$ CD117, a stem cell growth factor receptor encoded by the KIT gene, a receptor tyrosine kinase involved in cell survival and proliferation of melanocytes; CD271 (p75NTR), a neural crest stem cell factor, which has been shown to correlate with higher metastatic potential and worse clinical outcomes in melanoma; ${ }^{15}$ and Ki-67 protein, a marker of cellular proliferation, which is expressed during of all phases of cell cycling (G1, S, G2 and mitosis), but absent during the resting phase. ${ }^{16}$

\section{Materials and methods}

This study was approved by the Institutional Review Board of Boston Medical Center (H-30085). Reports of cases from the Skin Pathology Laboratory, Boston University School of Medicine, Boston, MA, between 2000 and 2010, with a diagnosis of desmoplastic melanoma were perused and 43 cases
(24 pure and 19 mixed desmoplastic melanomas) identified as meeting criteria for inclusion in this study with sufficient material for genetic and immunohistochemical analyses. Archival material from all 43 cases was retrieved. Histological sections of all cases were re-reviewed and the diagnoses confirmed. All patient data were de-identified. Tissue samples used were not selected for outcome measurements; hence, annotations regarding patient clinical data including follow-up/outcome are not included.

\section{Genetic Analyses}

DNA was extracted by proteinase $\mathrm{K}$ digestion of formalin-fixed, paraffin-embedded archival tissue. Direct DNA sequencing was performed on the $B R A F$ gene (forward gene coding strand only) spanning codon 600. RET G691S functional polymorphism was detected by direct DNA sequencing on the RET coding region on exon 11 with the forwarding primer. Sequencing of the KIT gene was performed using forward and reverse primers for coding regions of exons 11, 13 and 17 as described previously. ${ }^{17,18}$ Codon regions studied included the following: for exon 11, regions 550-591 (forward: $5^{\prime}$-TCCAGAGTGCTCTAATGAC-3' ${ }^{\prime}$, reverse: $5^{\prime}$-AGGTGGAACAAAACAAAGG-3'), for exon 13, regions 627-664 (forward: 5'-TACTGCATGCGCTTG ACATC-3', reverse: 5'-CCAAGCAGTTTATAATCT AGC-3') and for exon 17, regions 788-828 (forward: 5'-GTGAACATCATTCAAGGCGT-3' ${ }^{\prime}$, reverse: 5'-CCT TTGCAGGACTGTCAAGCA- $3^{\prime}$ ). Sequencing data were collected with ABI genetic analyzer 3130 and analyzed with ABI sequencing analysis software v5.3.1. For both $B R A F$ and RET sequencing, the reagents were ABI BigDye TerV3.1 cycle sequencing terminator ready reaction kit. Sequencing reactions were performed on an ABI 9700 thermocycler utilizing the ABI recommended protocol (Applied Biosystems, Foster City, CA, USA). A positive and/ or negative control was included in each batch of sequencing analysis.

\section{Immunohistochemical Analyses}

Sections of $5 \mu \mathrm{m}$ thick were obtained for immunohistochemical studies, which were performed on formalin-fixed, paraffin-embedded tissue, using standard peroxidase immunohistochemical techniques, heat-induced epitope-retrieval buffer and primary antibodies against Ki-67 (clone MIB-1; 1:150; Dako, Carpinteria, CA, USA), CD117 (NB120-956; 1:50; Novus Biologicals, Littleton, CO, USA), clusterin (anti-clusterin alpha chain, clone 41D; 1:150; Millipore, Billerica, MA, USA), nestin (MAB5326, 1:200, Millipore, Temecula, AZ, USA), SOX10 (1:200; Santa Cruz Biotechnology, Santa Cruz, CA, USA) and CD271 (p75NTR; clone EP1039Y, 1:100, Biocare Medical, LLC, Concord, 
CA, USA). Appropriate positive and negative controls were included. All stained slides were reviewed and scored by a dermatopathologist (MM) in a blinded fashion, to ensure consistency of interpretation.

Ki-67 nuclear reactivity was scored as a percentage on the basis of a manually performed 500-cell count in the most densely staining area in the dermis per previously established criteria. $^{19}$ For CD117, nestin, clusterin, SOX10 and CD271 (p75NTR), cut-off values for scoring were the following: negative or $0=<10 \%, 1=11-25 \%$, $2=26-50 \%$ and $3=>50 \%$. For purposes of statistical analyses, cases with $11 \%$ or more were considered positive.

\section{Statistical Analysis}

For analysis of direct DNA sequencing results, Fischer's exact test was used to compare categorical variables. For the results of immunostaining with Ki-67, CD117, Sox10 and p75NTR, Wilcoxon rank sums were used to compare means and s.d. of Ki-67 proliferation rates. Fisher exact test was used to compare the proportions of positivity to CD117, Sox 10 and p75NTR. All statistical analyses were performed with Statistical Analysis Software (SAS) version 9.2 (SAS Institute, Cary, NC, USA), and a $P$-value of less than 0.05 was considered statistically significant.

\section{Results}

\section{Clinical Data}

The demographics of the patients with pure and mixed desmoplastic melanoma are listed in Table 1 and compared in Table 2. Briefly, 22 patients were male and 19 were female, with no significant difference in gender distribution between the two

Table 1 Demographics of patients with pure (Cases 1-23) and mixed (Cases 24-41) desmoplastic melanoma

\begin{tabular}{|c|c|c|c|c|c|}
\hline Case number & Subtype & Age & Sex & Site & Clinically pigmented \\
\hline 1 & Pure & 68 & $\mathrm{~F}$ & $\mathrm{R}$ lower leg & Yes \\
\hline 2 & Pure & 47 & $\mathrm{M}$ & R cheek & Yes \\
\hline 3 & Pure & 81 & $\mathrm{M}$ & Sternal notch & Yes \\
\hline 4 & Pure & 62 & $\mathrm{~F}$ & R eyebrow & No \\
\hline 5 & Pure & 65 & $\mathrm{~F}$ & L orbital rim & No \\
\hline 6 & Pure & 67 & M & L great toe & Unknown \\
\hline 7 & Pure & 71 & $\mathrm{~F}$ & $\mathrm{R}$ post arm & Yes \\
\hline 8 & Pure & 55 & $\mathrm{~F}$ & L upper back & Yes \\
\hline 9 & Pure & 64 & M & Back & Yes \\
\hline 10 & Pure & 45 & M & $\mathrm{R}$ chest & No \\
\hline 11 & Pure & 74 & $\mathrm{M}$ & $\mathrm{R}$ lat arm & No \\
\hline 12 & Pure & 75 & $\mathrm{~F}$ & L nasal sidewall & No \\
\hline 13 & Pure & 68 & M & L ant neck & Yes \\
\hline 14 & Pure & 82 & $\mathrm{M}$ & Scalp & No \\
\hline 15 & Pure & 85 & M & L cheek & Yes \\
\hline 16 & Pure & 80 & M & L temple & No \\
\hline 17 & Pure & 81 & $\mathrm{M}$ & L hip & Yes \\
\hline 18 & Pure & 70 & $\mathrm{~F}$ & L dorsal hand & No \\
\hline 19 & Pure & 64 & $\mathrm{~F}$ & $\mathrm{R}$ arm & Yes \\
\hline 20 & Pure & 91 & M & Vertex scalp & Yes \\
\hline 21 & Pure & 77 & $\mathrm{~F}$ & L cheek & Yes \\
\hline 22 & Pure & 67 & M & Back & No \\
\hline 23 & Pure & 82 & $\mathrm{M}$ & R cheek & No \\
\hline 24 & Mixed & 91 & M & Vertex scalp & No \\
\hline 25 & Mixed & 67 & M & L cheek & Yes \\
\hline 26 & Mixed & 79 & $\mathrm{~F}$ & $\mathrm{R}$ forearm & No \\
\hline 27 & Mixed & 61 & $\mathrm{~F}$ & L upper back & No \\
\hline 28 & Mixed & 63 & M & $\mathrm{R}$ preauric & Yes \\
\hline 29 & Mixed & 84 & M & Mid-forehead & No \\
\hline 30 & Mixed & 38 & M & L lat cheek & No \\
\hline 31 & Mixed & 79 & $\mathrm{~F}$ & L leg & Yes \\
\hline 32 & Mixed & 71 & $\mathrm{~F}$ & $\mathrm{~L}$ arm & Yes \\
\hline 33 & Mixed & 16 & $\mathrm{~F}$ & $\mathrm{R}$ ear & No \\
\hline 34 & Mixed & 62 & M & L abdomen & Yes \\
\hline 35 & Mixed & 83 & $\mathrm{~F}$ & L shin & No \\
\hline 36 & Mixed & 92 & $\mathrm{~F}$ & Post scalp & No \\
\hline 37 & Mixed & 92 & $\mathrm{~F}$ & Post scalp & No \\
\hline 38 & Mixed & 92 & $\mathrm{~F}$ & $\mathrm{R}$ distal arm & Yes \\
\hline 39 & Mixed & 73 & M & $\mathrm{R}$ forearm & No \\
\hline 40 & Mixed & 77 & M & R ant scalp & Unknown \\
\hline 41 & Mixed & 61 & $\mathrm{~F}$ & L shoulder & No \\
\hline
\end{tabular}


study groups $(P=0.35)$. Patients with both subtypes each had an average age of 71 years at the time of diagnosis, and in both tumors presented in predominantly sun-exposed sites. A total of 20 of 41 were located on the head and neck, 13 of 41 on extremities, and 8 of 41 on the trunk; no significant difference in distribution was observed between the two groups. When available, data regarding clinically apparent pigmentation was recorded; 12 of 22 pure-type tumors were clinically suspected to be pigmented lesions, versus 6 of 17 mixed desmoplastic melanomas $(P=0.33)$.

\section{Histopathological Evaluation}

Tumors were classified as mixed desmoplastic melanoma according to the criteria established in multiple previous studies, ${ }^{4,20,21}$ in which the tumor contained two morphologically distinct populations of melanocytes with at least $10 \%$ of cells resembling conventional melanoma. Pure desmoplastic melanoma was defined as a predominantly spindled-cell tumor, with $>90 \%$ of tumor cells consistent with the typical desmoplastic melanoma morphology. Forty-three cases of desmoplastic melanoma were found in our records; of these, two cases represented re-excisions after initial biopsies, which were also identified on initial blinded search. Thus, a total 41 cases were included in the histological comparisons, of which 18 were classified as mixed (44\%) and 23 as pure desmoplastic melanoma (56\%). Histopathological features of the two groups are compared in Table 2. Average Breslow depth (for tumors not transected at their deep margin) was $4.2 \mathrm{~mm}$; mixed-type tumors had an average depth of $5.5 \mathrm{~mm} \quad(n=11$ cases $)$, whereas pure-type tumors averaged $3.74 \mathrm{~mm}(n=19$ cases; $P=0.76)$. Of 41 tumors, 28 contained a junctional component and 21 were pigmented microscopically, with no significant difference noted for the two groups for either

Table 2 Comparison of clinical and selected microscopic features of the two study groups. Only mitotic rate was significantly different between the two groups

\begin{tabular}{lcccc}
\hline & $\begin{array}{c}\text { All cases } \\
(\mathrm{n}=41)\end{array}$ & $\begin{array}{c}\text { Pure } \\
(\mathrm{n}=23)\end{array}$ & $\begin{array}{c}\text { Mixed } \\
(\mathrm{n}=18)\end{array}$ & P-value \\
\hline Average age & 71 & 71 & 71 & 0.59 \\
Male gender & 22 & 14 & 8 & 0.36 \\
Clinically pigmented & 18 & 12 & 6 & 0.33 \\
Breslow depth (mm) & 4.2 & $3.74^{\mathrm{b}}$ & $5.5^{\mathrm{c}}$ & 0.76 \\
Junctional component & 28 & 14 & 14 & 0.32 \\
Pigmentation & 21 & 12 & 9 & 1 \\
Neurotropism & 26 & 12 & 14 & 0.11 \\
Mitotic rate (per $\left.\mathrm{mm}^{2}\right)$ & 2.54 & 1.6 & 3.73 & 0.045
\end{tabular}

${ }^{\mathrm{a}}$ For one case from each subgroup, presence of pigmentation clinically was unknown.

${ }^{\mathrm{b}} \mathrm{A}$ total of 4 of 23 specimens were transected at their deep margin and were excluded.

${ }^{\mathrm{C}} \mathrm{A}$ total of 7 of 18 specimens were transected at their deep margin and were excluded. of these features. A statistically significant difference was observed for mitotic rate in the two groups; pure-subtype cases had an average of 1.6 mitoses per $\mathrm{mm}^{2}$, versus 3.73 per $\mathrm{mm}^{2}$ for mixed desmoplastic melanomas $(P=0.045)$.

\section{Genetic Analyses}

Polymorphism at the RET Coding Region (RETp) The results of genetic analysis and immunohistochemical staining are summarized in Table 3 and examples are shown in Figures 1 and 2. For genetic analysis, a total of 41 of the 43 initially identified cases were analyzed; for two of the initial group (both of the mixed subtype), the DNA extracted was suboptimal, precluding genetic analysis. In all, 12 of 41 of these cases (29\%) exhibited RETp mutations. Of 24 cases of pure desmoplastic melanoma, 8 (33\%) exhibited the G691S RETp, whereas in 16 (67\%), the polymorphism was absent. In 17 cases of mixed desmoplastic melanoma, RETp was present in 4 $(24 \%)$ and absent in $13(76 \%)$ cases; there was no statistically significant difference between the two groups $(P=\mathrm{NS})$.

\section{$B R A F$}

Of 41 cases tested, only $1 \quad(2 \%)$ exhibited BRAFV600E mutation; this was 1 of the 17 mixedsubtype cases $(6 \%)$. Of note, this case was negative for RETp. No pure-subtype cases exhibited BRAF mutation. There was no statistically significant difference between the two groups in this respect $(P=\mathrm{NS})$.

\section{KIT}

No mutations were identified in any of the 41 cases on analysis of exons 11, 13 and 17 of the $C$-KIT gene. Testing revealed wild-type genotype in all instances for all cases.

Table 3 Comparison of genetic and immunohistochemical profiles of the two groups

\begin{tabular}{|c|c|c|c|c|}
\hline & $\begin{array}{c}\text { All cases } \\
(\mathrm{n}=41)\end{array}$ & $\begin{array}{c}\text { Pure } \\
(\mathrm{n}=24)\end{array}$ & $\begin{array}{c}\text { Mixed } \\
(\mathrm{n}=17)\end{array}$ & $\mathrm{P}$-value \\
\hline \multicolumn{5}{|l|}{ Genetic analysis } \\
\hline RET polymorphism & 12 & 8 & 4 & NS \\
\hline BRAFV600E & 1 & 0 & 1 & NS \\
\hline \multirow[t]{2}{*}{ c-KIT mutation } & 0 & 0 & 0 & NS \\
\hline & $\begin{array}{c}\text { All cases } \\
(\mathrm{n}=41)\end{array}$ & $\begin{array}{c}\text { Pure } \\
(\mathrm{n}=23)\end{array}$ & $\begin{array}{c}\text { Mixed } \\
(\mathrm{n}=18)\end{array}$ & $\mathrm{P}$-value \\
\hline \multicolumn{5}{|l|}{ Immunohistochemistry } \\
\hline Ki-67\% & 15 & 5 & 28 & $<0.0001$ \\
\hline Positive CD117 & 20 & 6 & 14 & 0.002 \\
\hline Positive nestin & 35 & 19 & 16 & NS \\
\hline Positive Sox-10 & 37 & 20 & 17 & NS \\
\hline Positive clusterin & 2 & 1 & 1 & NS \\
\hline Positive CD271 & 26 & 14 & 12 & NS \\
\hline
\end{tabular}


a

GGT/AGT Poly
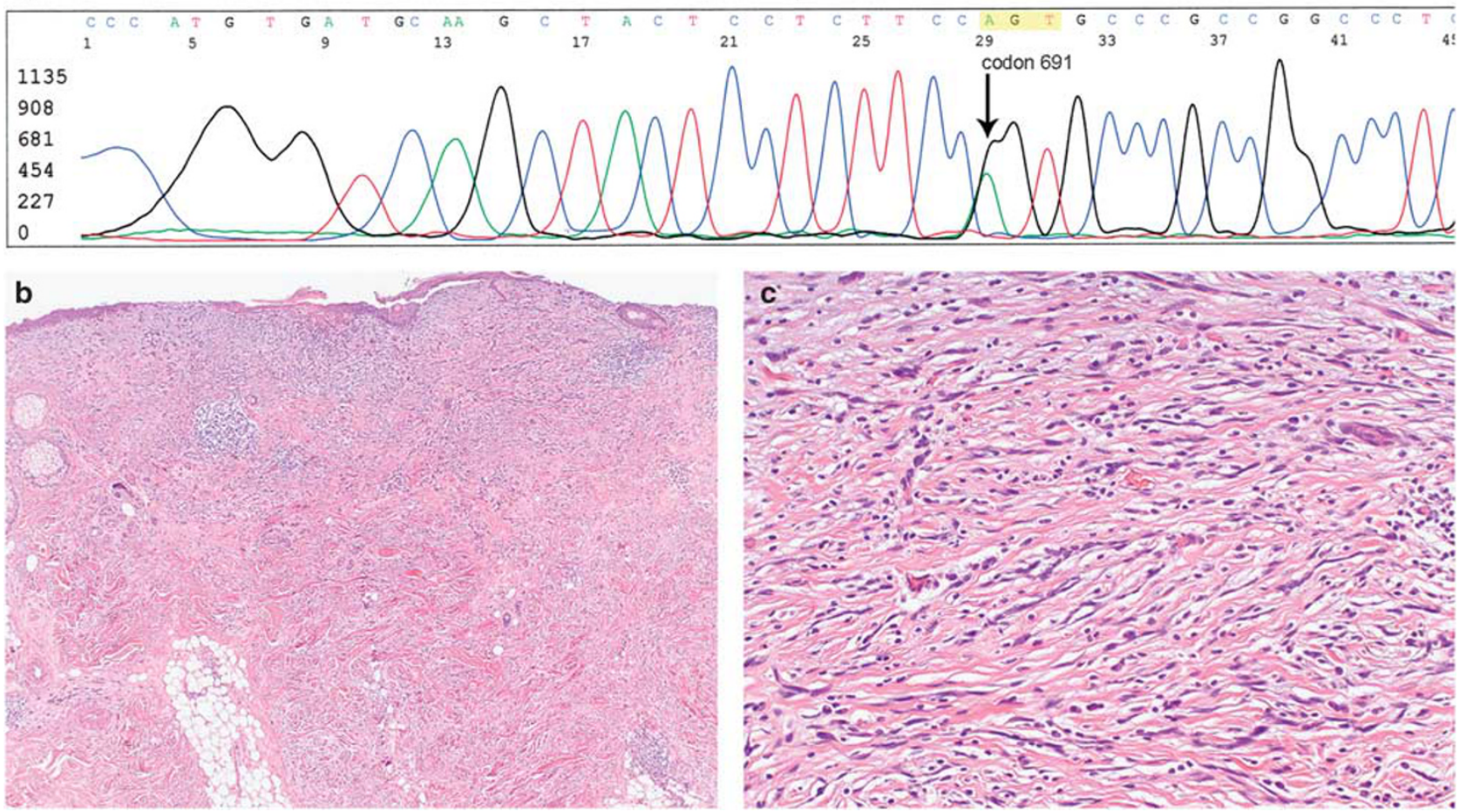

Figure 1 Pure desmoplastic melanoma (Case 16). (a) Results of direct DNA sequencing of exon 11 of the RET coding region, demonstrating RETp at codon 691 (GGT/AGT). (b) Hematoxylin-eosin stained section showing a diffuse infiltrate of spindled cells filling and expanding the dermis (low power). (c) High-power view, demonstrating a monomorphous spindle cell population (high power).

a

GGT/AGT Poly
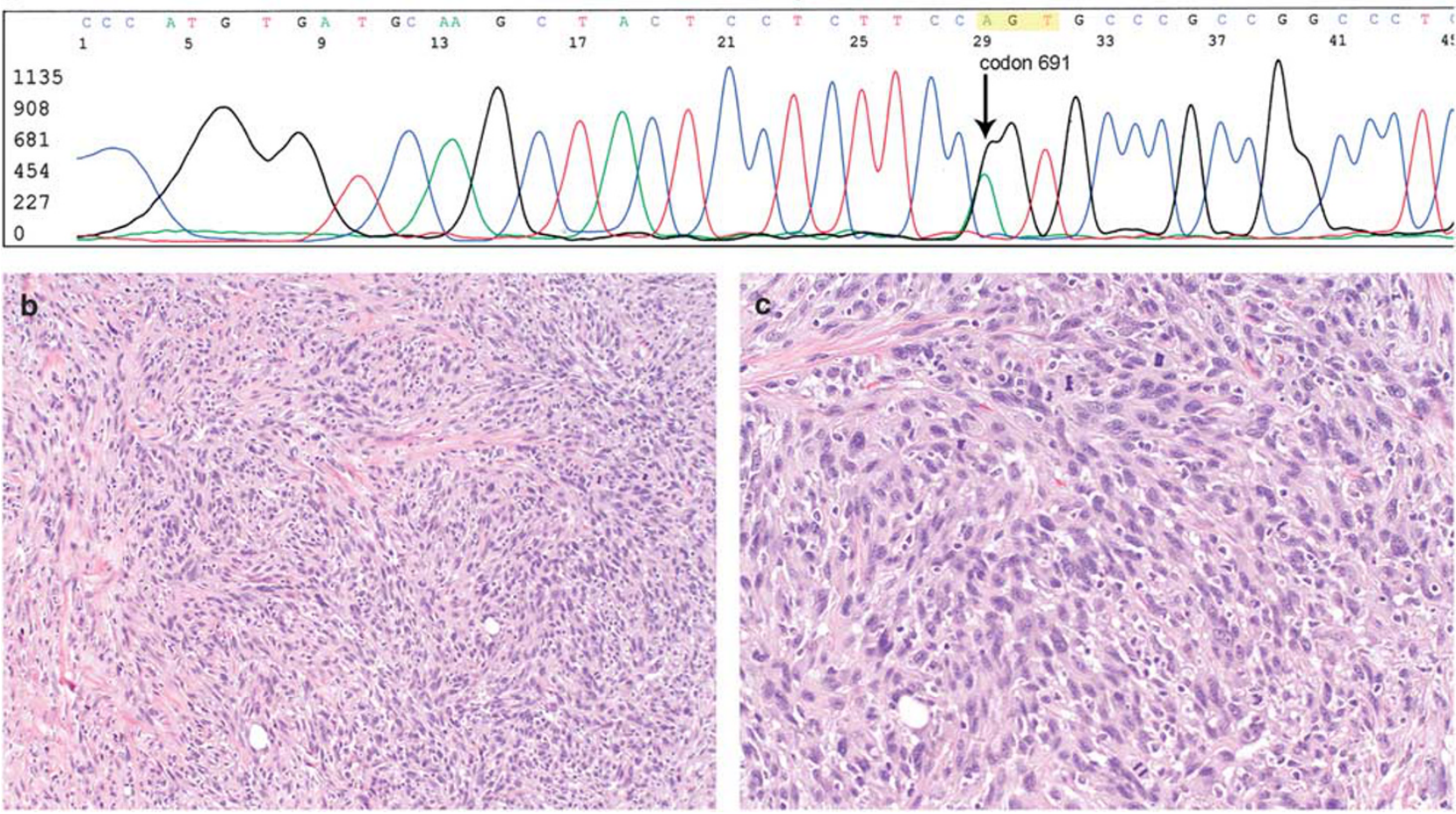

Figure 2 Mixed desmoplastic melanoma (Case 27). (a) Results of direct DNA sequencing, also with RETp at codon 691 (GGT/AGT).

(b) Hematoxylin-eosin stained section demonstrating a mixed population of spindled and epithelioid melanocytes (low power).

(c) High-power view, with a dense epithelioid cell population and multiple mitotic figures (high power). 


\section{Immunohistochemical Analyses}

Ki-67

Positive staining was noted by ascertaining positive nuclear staining, and any cytoplasmic staining was considered background artifact. Positive staining of cells in the basal layer of the epidermis and the follicular epithelium in cases where the same was visualized served as positive internal control. Mean percentage of Ki-67 nuclear positivity was 5\%
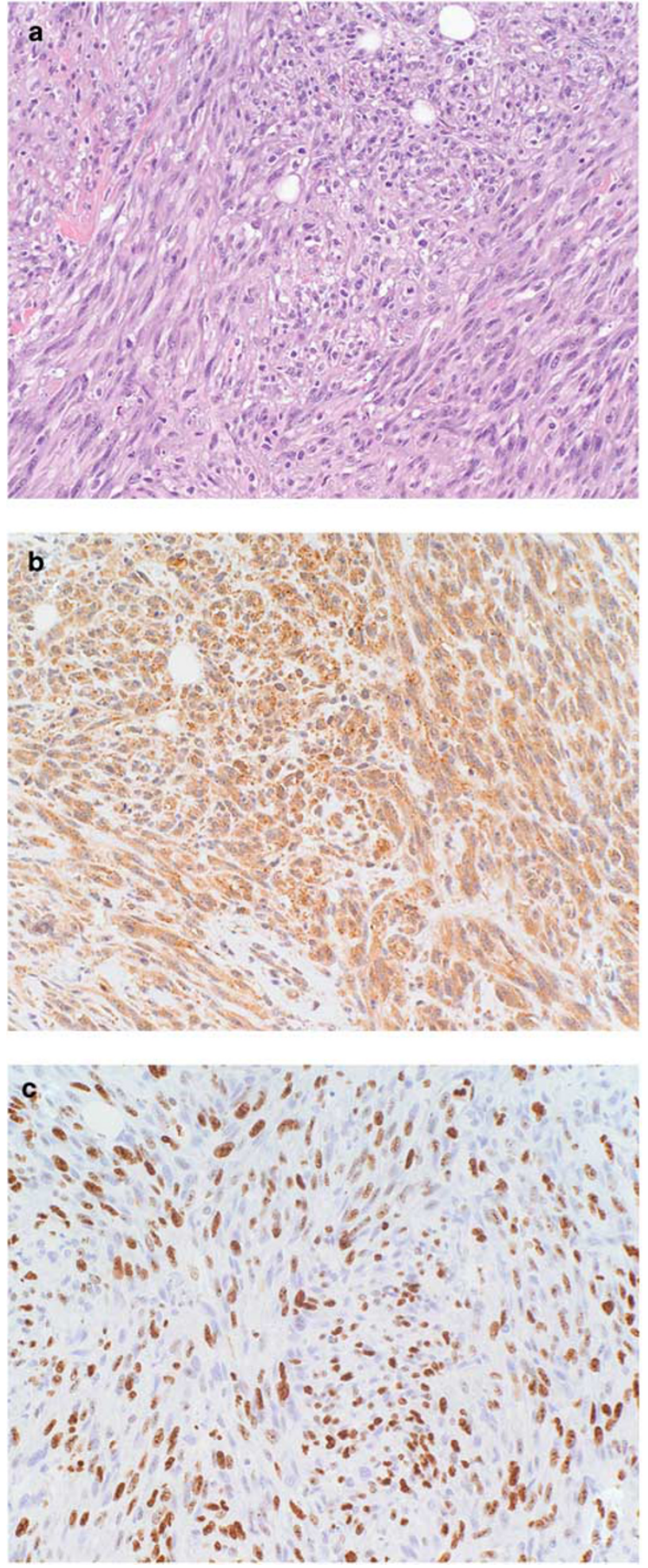
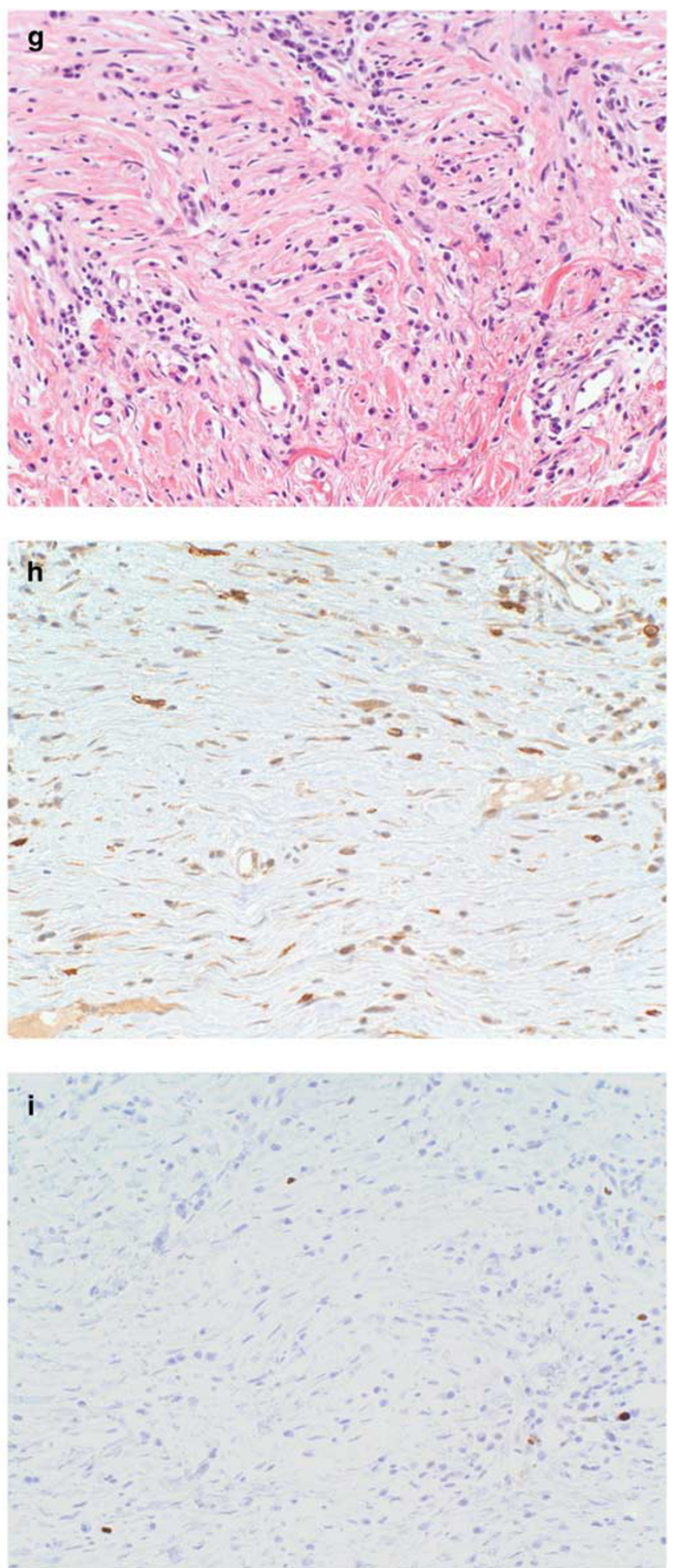

Figure 3 For Figure caption see next page. 

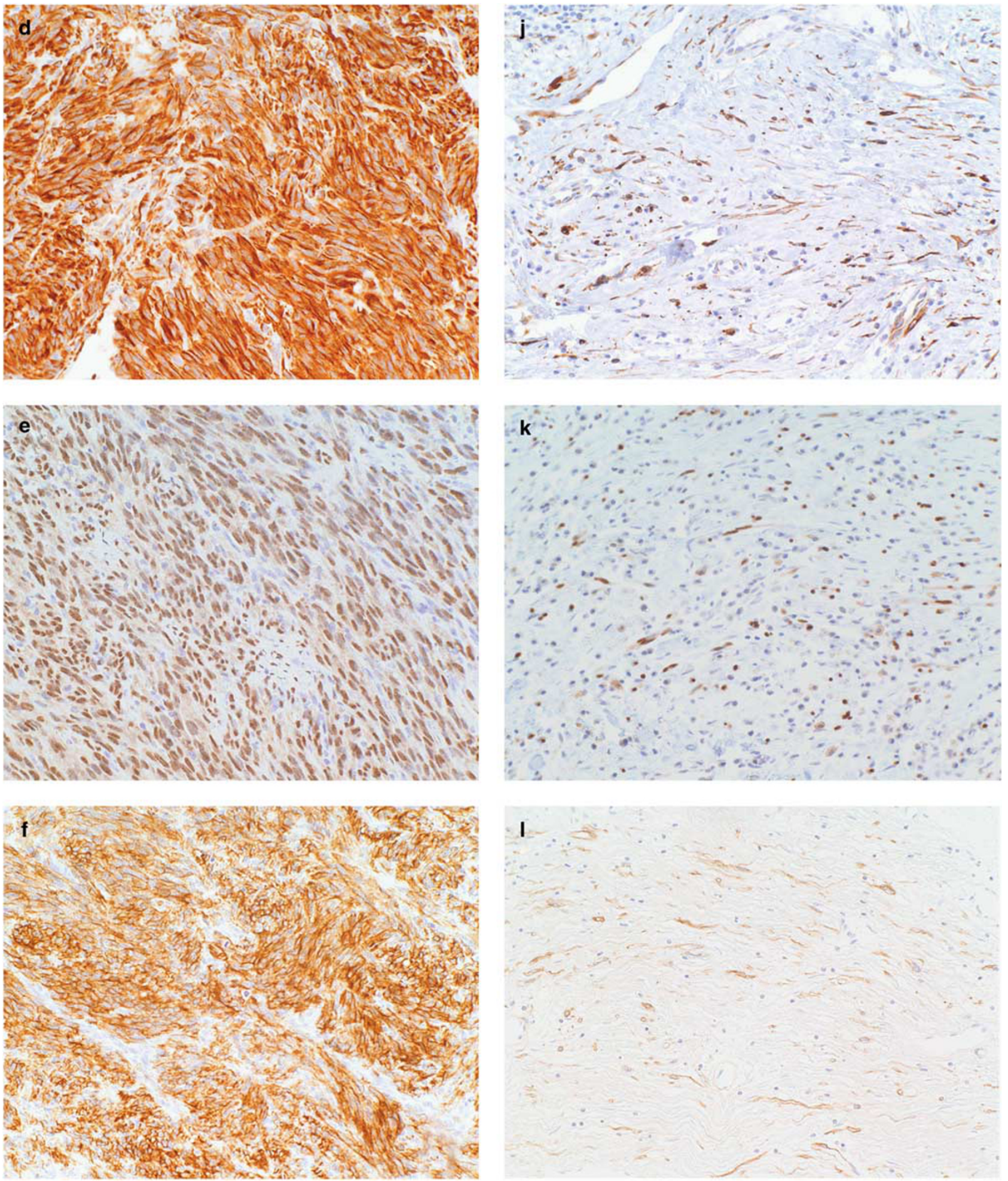

Figure 3 Immunohistochemical results of representative samples of mixed (a-f, Case 41) and pure (g-l, Case 22) desmoplastic melanoma. (a, g) Hematoxylin-eosin stained sections of mixed and pure tumors, respectively. (b, h) CD117 staining, with positive staining of both epithelioid and spindled cells in mixed desmoplastic melanoma. (c, i) Ki-67 staining. Proliferative index is markedly increased in the mixed-type case. (d, j) nestin, (e, k) SOX10 and (f, l) CD271 immunostaining, respectively.

$(\mathrm{s.d} .=6)$ in the pure subtype, versus $28 \%(\mathrm{s.d} .=12)$ in mixed-subtype tumors $(P<0.001)$. Figure 3 illustrates examples of immunohistochemical profiles of each tumor subtype.
CD117

Positive staining was noted by ascertaining cytoplasmic expression and any nuclear staining was considered the background artifact. Positive staining 

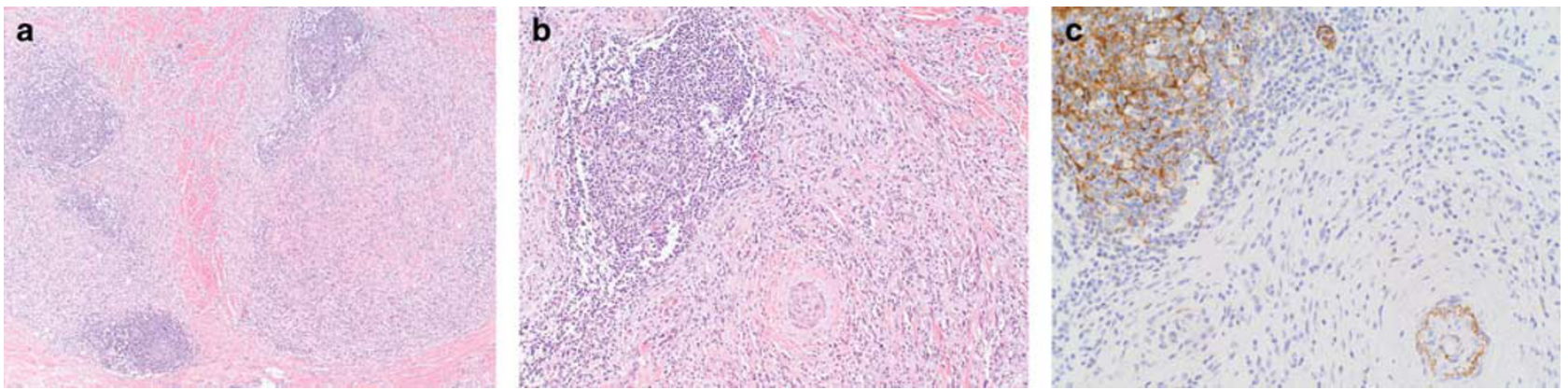

Figure 4 Mixed desmoplastic melanoma (Case 41), hematoxylin-eosin stained section (a; low power). (b) Focus of lymphocytic inflammation with germinal center formation (medium power). (c) Clusterin immunohistochemical stain, highlighting follicular dendritic cells, but without staining of lesional melanocytes (high power).

of melanocytes in the basal layer of the epidermis, basal layer of sebaceous glands, eccrine glands and mast cells (when present) served as the positive internal control. CD117 stained $26 \%$ (6 of 23 cases) of pure- versus $78 \%$ (14 of 18 cases) of mixed-subtype cases $(P<0.01)$, with equal intensity staining of both cell populations in the positive mixed-subtype cases.

\section{Nestin}

Positive staining was noted by ascertaining cytoplasmic expression and any nuclear staining was considered the background artifact. Positive staining of endothelial cells and/or in the inner aspect of the outer root sheath (below attachment of the pilar muscle) in cases where the same was visualized served as the positive internal control. Nestin stained $83 \%(19 / 23)$ of pure- versus $89 \%(16 / 18)$ of mixed-subtype cases $(P=\mathrm{NS})$.

\section{Clusterin}

Positive staining of elastic tissue fibers and follicular dendritic cells in cases where the same was visualized served as the positive internal control (Figure 4). Clusterin stained $4 \%(1 / 23)$ of pureversus $6 \%(1 / 18)$ of mixed-subtype tumors $(P=\mathrm{NS})$.

\section{SOX10}

Positive staining was noted by ascertaining nuclear expression and any cytoplasmic staining considered the background artifact. Positive staining of melanocytes in the basal layer of the epidermis and follicular epithelium in cases where the same was visualized served as the positive internal control. SOX10 stained $87 \%(20 / 23)$ of pure- versus $94 \%$ $(17 / 18)$ of mixed-subtype tumors $(P=\mathrm{NS})$.

\section{CD271 (p75NTR)}

Positive staining was noted by ascertaining cytoplasmic expression and any nuclear staining considered the background artifact. Positive staining of nerve fascicles, eccrine glands and the follicular epithelium (outer root sheath) in cases where the same was visualized served as the positive internal control. CD271 stained 61\% (14/23) of pure- versus $67 \%(12 / 18)$ of mixed-subtype tumors $(P=\mathrm{NS})$.

\section{Discussion}

Desmoplastic melanoma is an uncommon variant of cutaneous malignant melanoma; various estimates place its frequency between 1 and $4 \%$ of all diagnosed melanomas. ${ }^{21-23}$ Although desmoplastic melanoma was first characterized in the medical literature in $1971,{ }^{2}$ only recently (2004) has the concept of two morphological subtypes been put forth. ${ }^{20}$ Owing to the rarity of desmoplastic melanomas in general and this nascent classification system, the relative incidence of the two subtypes is not well established. Mixed desmoplastic melanoma appears to constitute between 40 and $60 \%$ of all cases obtained from histopathological archives, an estimate that appears to concur with findings from the current study in which $44 \%$ of cases $(18 / 41$ cases) were of the mixed subtype. ${ }^{4,22,24}$

The increased propensity for regional nodal metastasis of the mixed subtype has been previously established in several studies. ${ }^{4,25,26}$ In one study of approximately equal numbers of cases of pure and mixed desmoplastic melanomas, regional lymph node involvement was notably higher in the mixed subgroup (14 vs $3 \% ; P=0.02$ ), and overall 5 -year survival was significantly worse in this variant (61 vs $80 \%$; $P=0.001$ ). ${ }^{27}$ Furthermore, in the pure subtype cases, local recurrence was usually the first event, but in mixed desmoplastic melanoma, distant metastasis was usually the first event, without a preceding history of local recurrence. ${ }^{27}$ In another case series, systemic metastases appeared in 4 of 29 mixed desmoplastic melanoma patients $(14 \%)$ versus 1 of 26 with a pure-type tumor (4\%), and 5 patients with mixed-type tumors had diseaserelated death versus 2 with the pure type (although the authors did make note of the fact that the small sample size precluded any conclusions being drawn about the significance). ${ }^{22}$ In yet another series of patients with metastatic desmoplastic melanoma, the mixed subtype (hazard ratio 6.17; CI 1.61-23.81; 
$P=0.019)$ was an independent predictor of poorer overall survival in a multivariate analysis. ${ }^{28}$ Thus, the higher mortality of the mixed subtype appears to be well established. However, immunohistochemical and genetic differences between these groups have not been previously reported.

Early genetic studies revealed that desmoplastic melanoma does not typically demonstrate mutations in BRAFV600E, which is found in up to $60 \%$ of conventional melanomas. ${ }^{7}$ Our study further supports this observation, as only 1 of 41 cases exhibited $B R A F$ mutation. A more recent finding explains, in part, phenotypic properties of desmoplastic melanoma. ${ }^{10}$ Given that this variant is commonly neurotrophic, Narita et $a l^{10}$, investigated the rates of polymorphism in RET, which encodes a receptor tyrosine kinase for which the ligand is GDNF, and identified a statistically significant increased frequency of RETp mutation in desmoplastic versus conventional melanoma (61 vs 31\%). Functionally, mutations in RETp in cutaneous and non-cutaneous malignant tumors (including pancreatic cancer and melanoma) have been thought to alter responsiveness to GDNF and promote cellular proliferation via signaling through the RAS-BRAF-ERK, PI3K and $M A P K$ pathways. ${ }^{10,29,30}$ Of the 41 cases in the current study, 12 demonstrated mutations in RETp, though statistically significant differences in the frequency of RETp between pure and mixed desmoplastic melanoma $(8 / 24$ vs $4 / 17 ; P=\mathrm{NS})$ were not noted. Of the 12 cases, 10 (83\%) with RETp exhibited neurotropism microscopically, versus 17 of 29 cases (59\%) without RET polymorphism; although this difference did not reach statistical significance, there was a trend toward RETp being predictive of neurotropism $(P=0.165)$. The only case that exhibited the BRAF V599E mutation, a mixed-subtype tumor (Case 36), did not have a coexistent RETp mutation.

Immunohistochemistry results for CD117 were the most significant finding in the current study. In the only two previous studies documenting expression of CD117 in desmoplastic melanoma, the frequency of expression ranged from 21 to $29 \% .^{31,32}$ In the more recent of the two, CD117 stained the epidermal component in 8 of the 10 cases, in which 1 was present, and the dermal component in 3 of 14 cases $(21 \%) .{ }^{31}$ Although the subtypes were not specified, it seems reasonable to extrapolate that these cases were all of the pure variant, as all were described as 'clear cut' cases of desmoplastic melanoma. Frequency of CD117 expression in the pure subtype in our series (26\%) was similar to this finding.

Curtin et $a l^{12}$ found that melanomas with $c$-KIT mutation or copy number increase were significantly more likely to have a vertical growth phase component, and concluded that KIT expression is enhanced in tumors of more advanced stage (though none of the melanomas included their study were of desmoplastic type). We noted significantly enhanced expression of CD117 in 79\% of cases of the mixed subtype, with uniform staining of lesional cells in positive cases, with staining in both the spindled and 'epithelioid' components of the tumors. We observed no significant difference in either depth or mitotic rate for the positive and negative groups, though we suspect this may relate to the relatively small sample size. These findings should be validated by further studies on larger sample sizes of mixed desmoplastic melanoma cases.

When $c$-KIT, a transmembrane receptor tyrosine kinase, is activated via binding by its ligand (stem cell/steel factor), downstream events including activation of RAS/BRAF in the MAP kinase pathway are initiated, leading to cell proliferation. ${ }^{12,31}$ Given this, it makes sense that melanomas with $c$-KIT-activating mutations would not necessarily require $B R A F$ or $R A S$ mutations for oncogenesis. Desmoplastic melanoma would seem to share features in common with several melanoma types in this $C$-KIT group, including a predilection for sun-exposed sites and generally a lack of BRAF mutations. Head and neck locations are the most common sites for development of primary desmoplastic melanoma, ranging from 52 to $72 \%$ of all locations. ${ }^{22,33}$ Also of interest is the observation that in desmoplastic melanomas that have an associated intra-epidermal component, the majority exhibit a lentigo maligna-like phenotype (56 of 91 cases, $61 \%$ in one series; ${ }^{22}$ ), with generally small, monomorphous melanocytes in a lentiginous growth pattern and minimal pagetoid scatter. ${ }^{22,34,35}$ This also suggests homology with other melanomas on chronically sun-damaged skin, which commonly exhibit $c-K I T$ mutations. According to Curtin et al, ${ }^{12}$ 'melanoma types that have frequent genetic alterations of KIT typically have a lentiginous growth pattern, characterized by melanocytes lined up as single cells along the epidermis in the progression stage preceding invasive growth. By contrast, non-CSD [chronically sun exposed] melanomas, which have no KIT mutations or copy number increases, typically show a pagetoid growth pattern with melanocytes scattered throughout the epidermis.' Of the 14 cases, 11 (79\%) of CD117-positive mixed subtype had an overlying lentiginous junctional component, though this percentage was not significantly different than the CD117-negative mixed cases, nor the entire sample set as a whole (see Table 2).

We identified no mutations in $C$-KIT exons 11, 13 and 17 on genetic analysis of all tumors. Although the precise reasons for lack of correlation between genetic sequencing and c-KIT immunohistochemical expression are not clear, ours is not the first study to note lack of correlation. ${ }^{36,37}$ We recently observed a poor correlation between CD117 staining and $C$-KIT sequencing in atypical acral nevi. ${ }^{38}$ Briefly, we noted that CD117 stained $80 \%$ of acral nevi (with and without atypia), but genomic analyses of KIT exon regions 11,13 and 17 revealed no abnormalities in 'hotspots' frequently associated with point 
mutations in acral melanomas. ${ }^{38}$ One proposed theory is that the $c$-KIT product identified on positive immunostaining may be a non-mature protein rather than the active membranous receptor form. ${ }^{18}$ Another explanation may lie in the fact that copy number increases or gene amplifications may account for increased immunohistochemical expression without genetic mutations; ${ }^{39}$ copy number amplification of KIT can be involved in the upregulation of the MAP kinase pathway. Additionally, given that the coding region of the $c$-KIT gene is large, from a scientific perspective, it seems reasonable to speculate that a novel mutation that does not involve the examined exons, that is, 11, 13 and 17 may be responsible.

Busam et $a l^{11}$ have demonstrated that, compared with conventional melanoma, desmoplastic tumors show a significantly decreased expression of select genes related to melanin synthesis, and enhanced expression of multiple neurotrophic factors, observations consistent with the decreased pigmentation of desmoplastic melanoma, as well as its propensity for perineural invasion. ${ }^{21}$ In their initial screen, this group found that the gene coding for clusterin, believed to be involved in apoptotic pathways, was significantly increased in desmoplastic melanoma, with a 31-fold higher expression versus conventional melanoma on gene expression profiling. ${ }^{11}$ However, in a subsequent study, the same group noted that clusterin expression could only be identified in a minority of desmoplastic melanomas (7 of $21 ; 33 \%$ ) on immunohistochemical staining. ${ }^{40}$ We observed staining with clusterin in only two cases (one pure and one mixed variant). Although a possible explanation is that the results are reflective of differences relating to staining technique, both studies utilized the same clone of antibody, although dilutions were different. In addition, the scoring system incorporated here appears to be different from the one utilized by Busam et al, ${ }^{40}$ with different thresholds for positivity.

Additionally, we found a significantly increased Ki-67 proliferative rate in the mixed desmoplastic melanoma variant. Higher Ki-67 index has been associated with poorer overall and disease-free survival in cutaneous melanoma. ${ }^{41}$ Our finding here is similar to previous reports, in which higher mitotic rate was observed in mixed versus pure desmoplastic tumors, and correlated with more aggressive biological behavior. ${ }^{4,24}$

Nestin is an intermediate filament protein and marker for melanoma-initiating cells; immunohistochemical expression has been identified in desmoplastic melanoma ${ }^{42}$ and is associated with poor prognosis in conventional melanomas. ${ }^{13}$ CD271 (p75NTR) is a neural crest stem cell marker expressed in melanoma cells, which is associated with higher metastatic potential and worse prognosis. ${ }^{15}$ In the current study, nestin and CD271 stained 86 and $65 \%$, respectively, of all cases of desmoplastic melanoma with no statistically sig- nificant differences noted in the two variants, indicating that the populations of stem cells harbored appear to be similar in the two subtypes. Similar findings were obtained with respect to expression of SOX10, which stained 91\% of cases in the current series with no significant difference between the two subtypes. SOX10 is a transcription factor, which is important in melanocyte development. It is downregulated upon terminal differentation of melanocytes, and appears to be a necessary cofactor for the expression of nestin, which has been correlated with tumor progression. ${ }^{43-45}$ Given this, we hypothesized that SOX10 and/or nestin may have been differentially expressed in the two subtypes; the data did not support this theory.

A subclassification system of desmoplastic melanoma into pure and mixed subtypes has recently evolved on the basis of the clinical observation that these tumors behave in a heterogeneous fashion, some with limited lymph node metastasis and propensity for local recurrence, and others with higher nodal and distant metastasis and a poorer prognosis. In this study, although we noted many similarities between these two subtypes on genetic analysis and immunohistochemistry, we also noted a distinct difference in CD117 expression, which has not been previously recognized. This observation requires further study and validation, but may, in part, begin to explain the significant phenotypic differences between these two groups.

\section{Acknowledgement}

We are indebted to Dr Paolo Antonio Silva for his assistance with statistical analysis.

\section{Disclosure/conflict of interest}

The authors declare no conflict of interest.

\section{References}

1 Feng $\mathrm{Z}, \mathrm{Wu} \mathrm{X}$, Chen $\mathrm{V}$, et al. Incidence and survival of desmoplastic melanoma in the United States, 19922007. J Cutan Pathol 2011;38:616-624.

2 Conley J, Lattes R, Orr W. Desmoplastic malignant melanoma. Cancer 1971;28:914-916.

3 Baer SC, Schultz D, Synnestvedt M, et al. Desmoplasia and neurotropism. Prognostic variables in patients with stage I melanoma. Cancer 1995;76:2242-2247.

4 George E, McClain SE, Slingluff CL, et al. Subclassification of desmoplastic melanoma: pure and mixed variants have significantly different capacities for lymph node metastasis. J Cutan Pathol 2009;36:425-432.

5 Chin L. The genetics of malignant melanoma: lessons from mouse and man. Nat Rev Cancer 2003;3:559-570.

6 Chudnovsky Y, Adams AE, Robbins PB, et al. Use of human tissue to assess the oncogenic activity of melanoma-associated mutations. Nat Genet 2005;37: $745-749$. 
7 Davies H, Bignell GR, Cox C, et al. Mutations of the BRAF gene in human cancer. Nature 2002;417:949-954.

8 Brose MS, Volpe P, Feldman M, et al. BRAF and RAS mutations in human lung cancer and melanoma. Cancer Res 2002;62:6997-7000.

9 Davison JM, Rosenbaum E, Barrett TL, et al. Absence of V599E BRAF mutations in desmoplastic melanomas. Cancer 2005;103:788-792.

10 Narita N, Tanemura A, Murali R, et al. Functional RET G691S polymorphism in cutaneous malignant melanoma. Oncogene 2009;28:3058-3068.

11 Busam KJ, Zhao H, Coit DG, et al. Distinction of desmoplastic melanoma from non-desmoplastic melanoma by gene expression profiling. J Invest Dermatol 2005;124:412-419.

12 Curtin JA, Busam K, Pinkel D, et al. Somatic activation of KIT in distinct subtypes of melanoma. J Clin Oncol 2006;24:4340-4346.

13 Fusi A, Reichelt U, Busse A, et al. Expression of the stem cell markers nestin and CD133 on circulating melanoma cells. J Invest Dermatol 2011;131:487-494.

14 Ramos-Herberth FI, Karamchandani J, Kim J, et al. SOX10 immunostaining distinguishes desmoplastic melanoma from excision scar. J Cutan Pathol 2010;37: 944-952.

15 Civenni G, Walter A, Kobert N, et al. Human CD271positive melanoma stem cells associated with metastasis establish tumor heterogeneity and long-term growth. Cancer Res 2011;71:3098-3109.

16 Scholzen T, Gerdes J. The Ki-67 protein: from the known and the unknown. J Cell Physiol 2000;182: 311-322.

17 Chen LL, Trent JC, Wu EF, et al. A missense mutation in KIT kinase domain 1 correlates with imatinib resistance in gastrointestinal stromal tumors. Cancer Res 2004;64:5913-5919.

18 Torres-Cabala CA, Wang WL, Trent J, et al. Correlation between KIT expression and KIT mutation in melanoma: A study of 173 cases with emphasis on the acral-lentiginous/mucosal type. Mod Pathol 2009;22: 1446-1456.

19 Kapur P, Selim MA, Roy LC, et al. Spitz nevi and atypical Spitz nevi/tumors: a histologic and immunohistochemical analysis. Mod Pathol 2005;18:197-204.

20 Busam KJ, Mujumdar U, Hummer AJ, et al. Cutaneous desmoplastic melanoma: reappraisal of morphologic heterogeneity and prognostic factors. Am J Surg Pathol 2004;28:1518-1525.

21 Busam KJ. Cutaneous desmoplastic melanoma. Adv Anat Pathol 2005;12:92-102.

22 Soares de Almeida L, Requena L, Rutten A, et al. Desmoplastic malignant melanoma: a clinicopathologic analysis of 113 cases. Am J Dermatopathol 2008;30: 207-215.

23 Quinn MJ, Crotty KA, Thompson JF, et al. Desmoplastic and desmoplastic neurotropic melanoma: experience with 280 patients. Cancer 1998;83:1128-1135.

24 Murali R, Shaw HM, Lai K, et al. Prognostic factors in cutaneous desmoplastic melanoma. Cancer 2010;116: 4130-4138.

25 Pawlik TM, Ross MI, Prieto VG, et al. Assessment of the role of sentinel lymph node biopsy for primary cutaneous desmoplastic melanoma. Cancer 2006;106:900-906.

26 Hawkins WG, Busam KJ, Ben-Porat L, et al. Desmoplastic melanoma: a pathologically and clinically distinct form of cutaneous melanoma. Ann Surg Oncol 2005;12:207-213.

27 Maurichi A, Miceli R, Camerini T, et al. Pure desmoplastic melanoma: a melanoma with distinctive clinical behavior. Ann Surg 2010;252:1052-1057.

28 Murali R, Zannino D, Synnott M, et al. Clinical and pathological features of metastases of primary cutaneous desmoplastic melanoma. Histopathol 2011;58:886-895.

29 Takahashi M. The GDNF/RET signaling pathway and human diseases. Cytokine Growth Factor Rev 2001;12: 361-373.

30 Sawai H, Okada Y, Kazanjian K, et al. The G691S RET polymorphism increases glial cell line-derived neurotrophic factor-induced pancreatic cancer cell invasion by amplifying mitogen-activated protein kinase signaling. Cancer Res 2005;65:11536-11544.

31 North J. CD117 (c-KIT) staining in desmoplastic melanoma. J Cutan Pathol 2011;38:753-755.

32 Winnepenninckx V, De Vos R, Stas $\mathrm{M}$, et al. New phenotypical and ultrastructural findings in spindle cell (desmoplastic/neurotropic) melanoma. Appl Immunohistochem Mol Morphol 2003;11:319-325.

33 Lens MB, Newton-Bishop JA, Boon AP. Desmoplastic malignant melanoma: a systematic review. Br J Dermatol 2005;152:673-678.

34 Egbert B, Kempson R, Sagebiel R. Desmoplastic malignant melanoma. A clinicohistopathologic study of 25 cases. Cancer 1988;62:2033-2041.

35 Carlson JA, Dickersin GR, Sober AJ, et al. Desmoplastic neurotropic melanoma. A clinicopathologic analysis of 28 cases. Cancer 1995;75:478-494.

36 Kong Y, Si L, Zhu Y, et al. Large-scale analysis of KIT alterations in Chinese patients with melanoma. Clin Cancer Res 2011;17:1684-1691.

37 Beadling C, Jacobson-Dunlop E, Hodi FS, et al. KIT gene mutations and copy number in melanoma subtypes. Clin Cancer Res 2008;14:6821-6828.

38 Park E, Yang S, Emley A, et al. Lack of correlation between immunohistochemical expression of CKIT and KIT mutations in atypical acral nevi. Am J Dermatopathol; advance online publication, 15 November 2011 (e-pub ahead of print).

39 Sihto H, Sarlomo-Rikala M, Tynninen O, et al. KIT and platelet-derived growth factor receptor alpha tyrosine kinase gene mutations and KIT amplifications in human solid tumors. J Clin Oncol 2005;23:49-57.

40 Busam KJ, Kucukgol D, Eastlake-Wade S, et al. Clusterin expression in primary and metastatic melanoma. J Cutan Pathol 2006;33:619-623.

41 Henrique R, Azevedo R, Bento MJ, et al. Prognostic value of Ki-67 expression in localized cutaneous malignant melanoma. J Am Acad Dermatol 2000;43:991-1000.

42 Shimada S, Tsuzuki T, Kuroda M, et al. Nestin expression as a new marker in malignant peripheral nerve sheath tumors. Pathol Int 2007;57:60-67.

43 Cook AL, Smith AG, Smit DJ, et al. Co-expression of SOX9 and SOX10 during melanocytic differentiation in vitro. Exp Cell Res 2005;308:225-235.

44 Flamminger A, Besch R, Cook AL, et al. SOX9 and SOX10 but not BRN2 are required for nestin expression in human melanoma cells. J Invest Dermatol 2009;129:945-953.

45 Brychtova S, Fluraskova M, Hlobilkova A, et al. Nestin expression in cutaneous melanomas and melanocytic nevi. J Cutan Pathol 2007;34:370-375. 